\title{
Book Review: Marcus Callies and Sandra Götz (eds.). (2015). Learner corpora in language testing and assessment (Studies in Corpus Linguistics 70) Amsterdam/ Philadelphia: John Benjamins, pp 220.
}

\author{
Jesús García Laborda ${ }^{1}$ \\ Universidad de Alcalá de Henares
}

El estudio de corpus lingüísticos es antiguo. Sin embargo, los estudios de corpus en exámenes y evaluaciones se han centrado en aquellos segmentos discursivos con errores con el fin de promover su revisión y posterior omisión. Por tanto, es un enfoque excesivamente pragmático que, sin embargo, no permite un desarrollo competente del discurso del estudiante. En este sentido, este libro es un excelente paso adelante hacia un uso realmente influyente para los estudios en lingüística aplicada. Learner Corpora in Language Testing and Assessment es el volumen 70 de la colección Studies in Corpus Linguistics (SCL) (John Benjamins) coeditado por Marcus Callies y Sandra Götz, que incluye una introducción sobre el tema del libro seguido de una selección de ocho trabajos basados en un taller previo al 34 Congreso Internacional ICAME celebrado en Santiago de Compostela (España) del 22 al 26 de mayo de 2013 con el mismo tema que este libro.

El propósito de los autores es mostrar los beneficios y el potencial de usar corpus de los estudiantes para el desarrollo de las competencias orales y escritas mostrando los beneficios de este nuevo campo a caballo entre la evaluación lingüística y el estudio de la lingüística de corpus dándole, de esta manera, un sitio propicio en los estudios de lingüística aplicada al tiempo que busca "for a better operationalization and control of the construct of L2 proficiency" (p. 1). No cabe duda de que la competencia necesita ser definida en muchos exámenes para conseguir la validación en la generalización de los resultados y, por tanto, en la validez de impacto. De aquí

Corresponding author - Universidad de Alcalá de Henares, Departamento de Filología Moderna, Madrid (Spain). Email: jesus.garcialaborda@uah.es 
surge la necesidad de describir detalladamente descriptores y detalles adecuados que delimiten claramente los descriptores de competencia ya sea las directrices ACTFEL o CEFR que siempre han sido difíciles de delimitar.

El capítulo introductorio del libro sirve para definir y explicar cómo se obtienen los datos. Seguidamente explica las cuestiones metodológicas que conforman el volumen. La primera se relaciona con el hecho de que las evaluaciones generalmente se hacen en relación y comparación con una segmentación por niveles basada en un hablante nativo competente y articulado usuario ideal de la lengua que se obtienen a partir del Cambridge Learner Corpus, por ejemplo. El segundo aspecto metodológico a la obtención de datos para incrementar los corpus y poder delimitar más claramente los segmentos (o niveles) citados. El tercer aspecto mencionado en el libro se relaciona con las diferencias individuales que, sin duda, aportan datos útiles a la hora de delimitar los niveles de un examinado ideal.

En relación a los capítulos, lo primero que llama la atención es calidad misma de los autores. El libro, se divide en dos partes, la primera se relaciones con nuevos recursos, herramientas y métodos para el análisis de corpus en este contexto diseñadas y / o desarrolladas por los propios autores. En esta primera parte Rolf Kreyer presenta un nuevo corpus, específicamente, el Marburg Corpus of Intermediate Learner English (MILE) actualmente en progreso realizado con estudiantes de los grados 9 al 12 en escuelas secundarias en Alemania. Dicho corpus está pensado para mostrar el progreso de los estudiantes en sus últimos años de escuela secundaria. Dicho trabajo es muy necesario ya que son muy pocos los estudios que ilustran el avance de los estudiantes y que añade un dinamismo que es necesario para nuevos tipos de evaluación, especialmente en la tendencia de la Evaluación Dinámica de las lenguas. Seguidamente Gamallo, García, Del Río y González muestran una herramienta para detectar y clasificar errores en lengua gallega escrita a través de procesamiento de lenguaje natural. El tercer capítulo de esta parte, de Nordrum y Eriksson, presentan su enfoque a través de protocolos verbales obtenidos de trabajos académicos de corrección en materiales audiovisuales mostrando la autoevaluación formativa puede ser desarrollada a través de actividades realizadas por ordenador basadas en un corpus. Esta primera parte se cierra con el artículo de Merchand y Akutsu sobre un método para asignar niveles de competencia a textos comunicativos mediados por ordenador a un corpus obtenido de comentarios en artículos de noticias.

Los capítulos de la sección dos se basan en enfoques inteligentes para la evaluación de la competencia lingüística. En esta parte hayamos capítulos con metodologías innovadoras que muestran como la competencia puede ser operativa a partir de datos obtenidos de corpus a través de la identificación centrada en el texto y usando descriptores basados en la obtención de datos de los distintos tipos de estudiantes. 
Estos datos también serán utilizados para la corrección humana. Así, en el capítulo cinco, Agnieszka Leńko-Szymańska explora la utilidad del English Vocabulary Profile (EVP). Así, analizando 90 ensayos obtenidos del International Corpus of Crosslinguistic Interlanguage (ICCI), que se ajusta al CEFR, observa la utilidad y la validez externa de los descriptores EVP por su alta correlación con el CEFR a través de la asignación por evaluadores humanos. El excelente trabajo de Pascual Pérez-Paredes and María Sánchez-Tornel perfile las figuras del componente descriptivo del Louvain International Database of Spoken English Interlanguage (LINDSEI) a través de su comparación con el Louvain Corpus of Native English Conversation (LOCNEC)usando una versión adaptada de análisis multidimensional de Biber. Su estudio muestra que los estudiantes de inglés como lengua extranjera representadas en el enfoque LINDSEI operan de manera muy diferente que los hablantes nativos en término de frecuencia de uso de varias características lingüística. Esto puede tener unas implicaciones interesantes mostrando el por qué los estudiantes evaluados podrían mostrar alternancias en evaluaciones orales que discrepan claramente de las de los hablantes nativos con un efecto intrínseco en la validez de la corrección de los ejercicios de evaluación, especialmente en la evaluación formal. Otra de las grandes investigadoras españolas en corpus aplicado a la evaluación, María Belén Díez-Bedmar presenta un estudio piloto basado en un corpus que combina las medidas de frecuencia y las de corrección para explorar el uso del artículo por estudiantes de inglés españoles con niveles A2, B1 y B2 del CEFR. Su trabajo puede ser útil para evaluadores de exámenes. El cuarto capítulo de esta parte por Sandra Götz adopta un método basado en corpus sobre la calidad de la producción oral de estudiantes avanzados de alemán e informa sobre sus descubrimientos sobre errores de tiempo verbal (especialmente en estilo indirecto y condicionales) en el componente en alemán del LINDSEI. Su estudio muestra de manera un tanto sorprendente que los estudiantes de nivel avanzado parecen ser muy heterogéneos en referencia al número y tipo de errores que cometen aunque sea de manera sistemática lo que permite describir los tipos y características de los errores de este nivel. Aunque esto parece realmente aplicable en alemán donde no hay grandes diferencias entre registros y dialectos en comparación al inglés. Por otro lado, aunque no se ha planteado en estudios previos, podría ser que los hablantes nativos también usen un tipo de interlenguaje hacia una versión "correcta" universal que trascienda a distintos registros y dialectos que justificaría los usos y errores también sistemáticos encontrados en los hablantes nativos. Por tanto, más que servir para categorizar la producción de los hablantes no nativos este análisis de errores muestra la sistematicidad que se encuentra en hablantes avanzados y también hablantes nativos.

Sin ser un experto en el uso de corpus, se me hizo evidente inmediatamente varias cuestiones positives del libro: primero, su notable utilidad en el campo de la evaluación tanto para administradores como para usuarios a través de potenciales 
aplicaciones prácticas de estos estudios; segundo, el uso de corpus es necesario para la mejora de aplicaciones informáticas tales como e-rater u otros sistemas similares. En resumen, este es un volumen más que bienvenido en la investigación en evaluación con un valor muy alto internacional especialmente desde una perspectiva metodológica. Se trata de un libro cuyo único problema es que debe ser seguido por estudios posteriores, quizás por estos mismos autores. En conclusión, este volumen puede y debería llegar a ser un referente en los estudios sobre validez interna, diseño de rúbricas, estudios sobre el CEFR y los aspectos evolutivos de la formación específica para exámenes.

\section{About the author}

Dr. Jesús García Laborda es profesor titular de Filología Inglesa especializado en tecnología de la enseñanza de ESP y exámenes de Alto impacto. Tiene más de 90 publicaciones indexadas en el Web of Knowledge. En la actualidad es probablemente uno de los revisores de libros más significativos de España (con más de 50 Book reviews en JCR-ISI) incluyendo Language Testing, ESP Journal, British Journal of Educational Technology o Modern Language Journal. 